\title{
Primary Rosai-Dorfman disease of bone arising in the infantile ilium: A case report
}

\author{
YUYA IZUBUCHI $^{1}$, KOJI SUZUKI ${ }^{2}$, YOSHIAKI IMAMURA ${ }^{3}$, HAJIME KATAYAMA ${ }^{4}$, \\ YUSEI OHSHIMA ${ }^{2}$ and AKIHIKO MATSUMINE ${ }^{1}$
}

\author{
${ }^{1}$ Department of Orthopaedics and Rehabilitation Medicine, Unit of Surgery, Division of Medicine, \\ Faculty of Medical Sciences, University of Fukui; ${ }^{2}$ Department of Pediatrics, Faculty of Medical Sciences, \\ University of Fukui; ${ }^{3}$ Division of Diagnostic Pathology/Surgical Pathology, University of Fukui Hospital, Fukui 910-1193; \\ ${ }^{4}$ Katayama Orthopaedic Clinic, Monjyu Medical Corporation, Fukui 918-8016, Japan
}

Received October 13, 2019; Accepted January 29, 2020

DOI: $10.3892 / \mathrm{etm} .2020 .8568$

\begin{abstract}
Rosai-Dorfman disease (RDD) is an extremely rare benign histiocytic disorder that usually affects young adults. Extranodal involvement of the RDD is common and may occur in $>40 \%$ of patients, but bone involvement occurs in $<10 \%$ of cases. Furthermore, primary bone RDD is extremely rare. The present study reports a case of primary bone RDD arising in the infantile ilium. Plain radiographs and computed tomography (CT) revealed an osteolytic lesion at the peri-acetabular region of the patient's right ilium. Fluorodeoxyglucose positron emission tomography indicated an abnormal accumulation only in the right iliac bone, without any other accumulation. An open biopsy was performed and the diagnosis of primary RDD of bone in the ilium was made. The bone lesion exhibited spontaneous regression on radiography, and the patient was able to walk without any limping or pain at 8 months after the biopsy. After 18 months of follow-up, the bone lesion had completely disappeared, and no joint deformity was observed on radiography or CT. The present report described the clinicopathological details of this rare case and reviewed the relevant literature.
\end{abstract}

\section{Introduction}

Rosai-Dorfman disease (RDD) is a benign histiocyte disorder that was originally described by Rosai and Dorfman in 1969 as sinus histiocytosis with massive lymphadenopathy. It mostly affects young adults. Patients typically present with a fever, leukocytosis, and non-painful cervical lymphadenopathy $(1,2)$.

Correspondence to: Professor Akihiko Matsumine, Department of Orthopaedics and Rehabilitation Medicine, Unit of Surgery, Division of Medicine, Faculty of Medical Sciences, University of Fukui, 23-3 Matsuoka-Shimoaizuki, Eiheiji-Cho, Yoshida-gun, Fukui 910-1193, Japan

E-mail: matsumin@u-fukui.ac.jp

Key words: Rosai-Dorfman disease, primary, bone, infant
RDD sometimes involves extranodal organs, including the skin, soft tissue, and central nervous system (1). Bone involvement occurs in less than $10 \%$ of cases. Primary bone RDD, in the absence of lymphadenopathy, accounts for less than $1 \%$ of all cases (2). RDD is usually self-limiting disease, making systemic therapy rarely required (3). The imaging of osseous RDD typically shows a lytic lesion on radiography and CT. The differential diagnosis is broad and includes osteomyelitis, Langerhans cell histiocytosis, lymphoma, primary bone sarcoma and metastatic bone tumor (4). Therefore accurate diagnosis depends on histological examination.

We herein report a rare case of primary bone RDD that occurred in the pelvic bone of a two-year-old boy and we present a literature review of the management and clinical course of this type of disease.

\section{Case report}

A two-year-old boy was admitted to our hospital due to limping of the right lower extremity, which persisted for two months without obvious pain. He had no perinatal medical problems. He had no history of the infection, such as upper respiratory tract infection or viral enteritis.

A physical examination showed slight limitation in the range of motion of his right hip joint without a leg length discrepancy. There was no swelling, redness, local heat, or percussion pain around his right hip joint. There was no cervical, axillary, popliteal, or inguinal lymphadenopathy. A laboratory examination revealed no abnormalities; his white blood cell count and hemoglobin and serum C-reactive protein (CRP) levels were normal. Tests for tumor markers, including AFP, CEA, CA19-9, CA125, NSE, HCG- $\beta$ and sIL-2R, were negative.

Plain radiography showed an osteolytic lesion at the peri-acetabular region of his right ilium (Fig. 1). Computed tomography (CT) showed a purely osteolytic lesion of the right ilium with slight discontinuity of the thin cortical bone and minor cortical fracture (Fig. 2). New bone formation was not observed. Magnetic resonance imaging of the pelvis showed a peri-acetabular lesion at the ilium with an iso- to high heterogeneous signal intensity on T1-weighted imaging and a high heterogeneous signal intensity on $\mathrm{T} 2$-weighted 


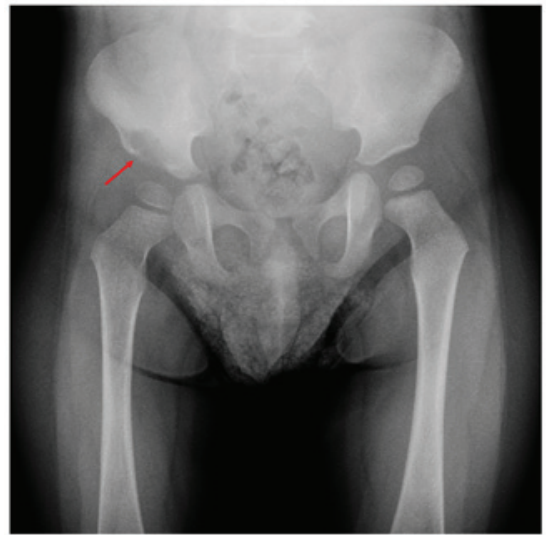

Figure 1. Plain radiography at first medical examination. Radiograph showing an osteolytic lesion of the right acetabulum (arrow). No significant periosteal reaction was observed.
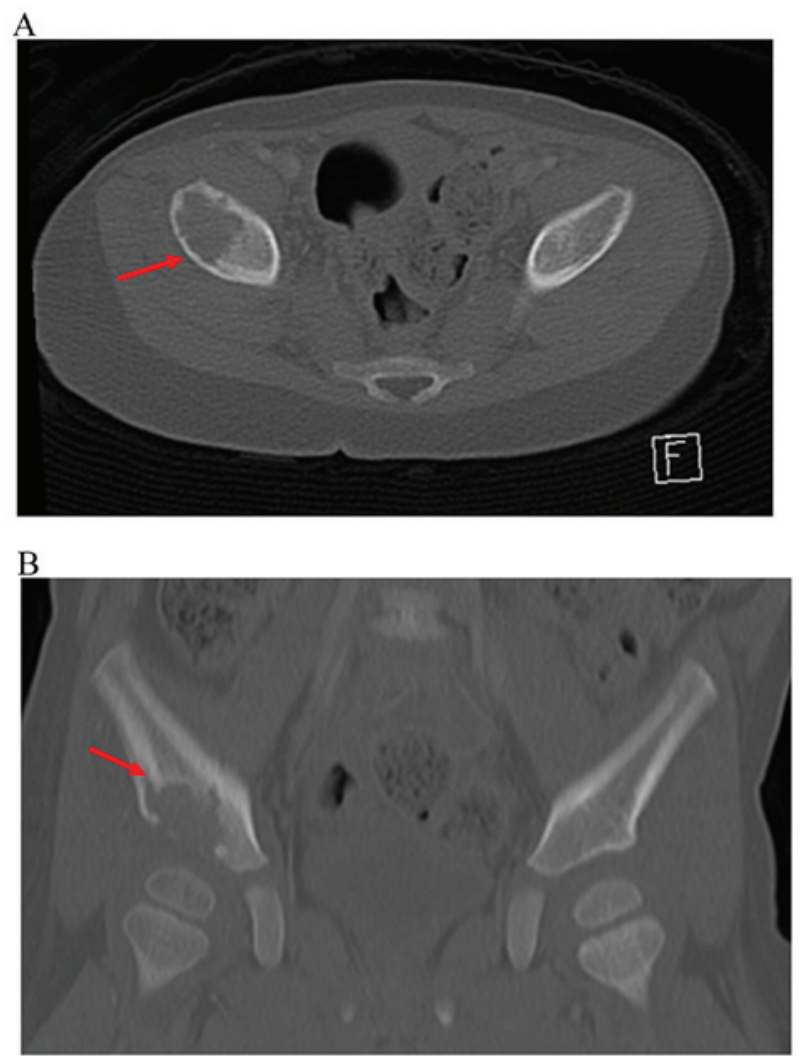

Figure 2. CT images of pelvis. (A) Axial CT image showing the purely osteolytic lesion of the right ilium (arrow). (B) Coronal CT image showing discontinuity of the thin cortical bone (arrow). New bone formation and periosteal reaction were not observed.

imaging (Fig. 3). Fluorodeoxyglucose positron emission tomography (FDG-PET) showed an abnormal accumulation in the right peri-acetabular lesion.

An incisional biopsy was performed to obtain a definite pathological diagnosis. Microscopy showed numerous large histiocytes interspersed with different amounts of lymphocytes, neutrophils, and plasma cells. Emperipolesis was observed in the cytoplasm of the large histiocytes. Immunohistochemistry was positive for CD68, CD163 and S-100 and negative for CDla (Fig. 4). The patient was diagnosed with primary RDD of bone in the ilium. Because the osteolytic lesion gradually
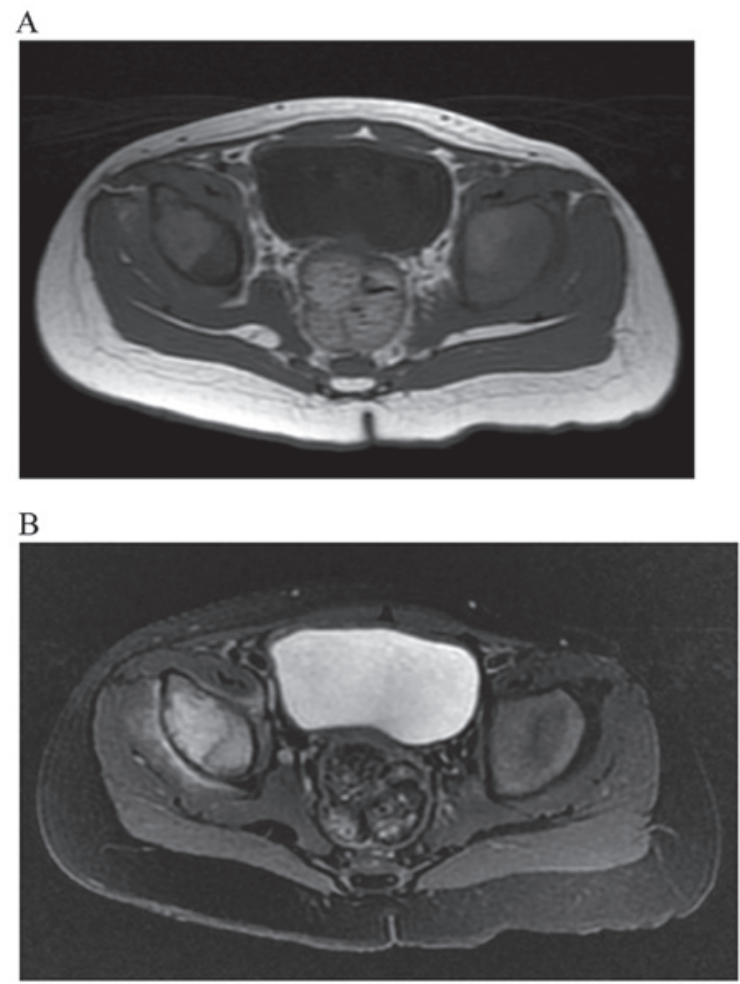

Figure 3. MR images of pelvis. MR images showing the peri-acetabular lesion of the ilium with (A) iso- to high heterogeneous signal intensity on T1-weighted images and (B) high heterogeneous signal intensity on T2-weighted images.

diminished without any progression of clinical symptoms after the excisional biopsy, we carefully observed the patient without additional treatment. After 18 months of follow-up, the bone lesion on radiography and CT had disappeared completely, and no joint deformity was observed (Fig. 5).

We are concerned about the risk of recurrence, growth failure, and osteoarthritis. We are planning to follow him until he reaches adulthood.

\section{Discussion}

RDD is a rare histiocytic disorder initially described as a separate entity in 1969 by Rosai and Dorfman under the term sinus histiocytosis with massive lymphadenopathy (1). The majority of patients are adolescents and young adults, and the mean age at the onset is 20 years old. The analysis of a registry of 423 worldwide cases of RDD showed that the mean age of the onset was 20.6 years, with $58 \%$ of cases occurring in men and $42 \%$ in women (2).

The pathological findings of RDD are characterized by the proliferation of numerous large histiocytes containing abundant eosinophilic cytoplasm, enmeshed in a variably cellular, mixed inflammatory infiltration composed of plasma cells, lymphocytes, neutrophils, foamy macrophages and rare eosinophils. The characteristic feature of the large histiocytes in RDD is conspicuous emperipolesis-namely lymphocytophagocytosis-with intracytoplasmic lymphocytes, plasma cells, or neutrophils $(3,5)$. The histiocytes of RDD are immunoreactive for CD68, CD163 and S100 protein and lack reactivity for CD1a (3). This immunohistochemical feature 


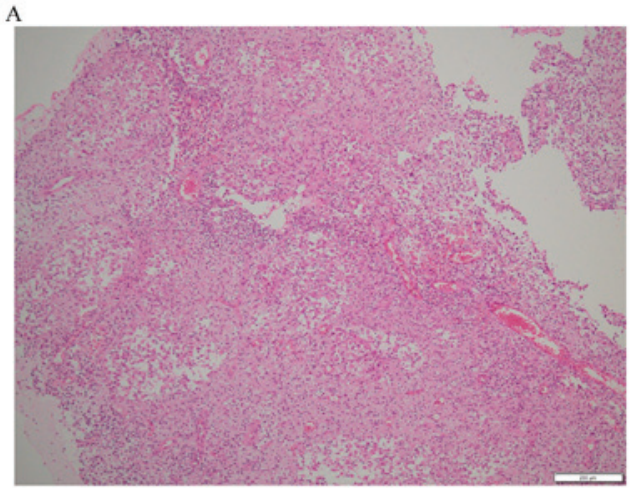

C

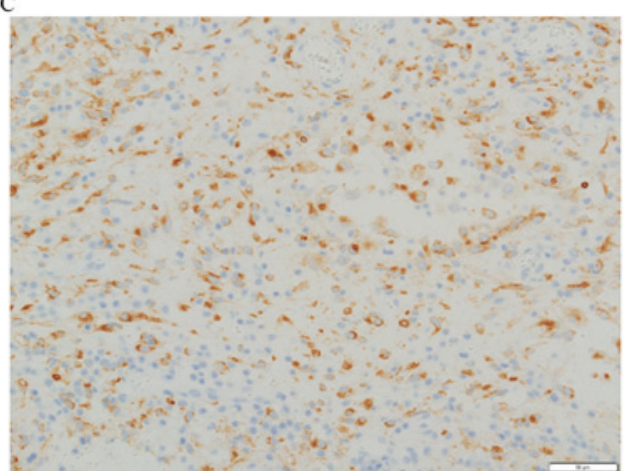

B

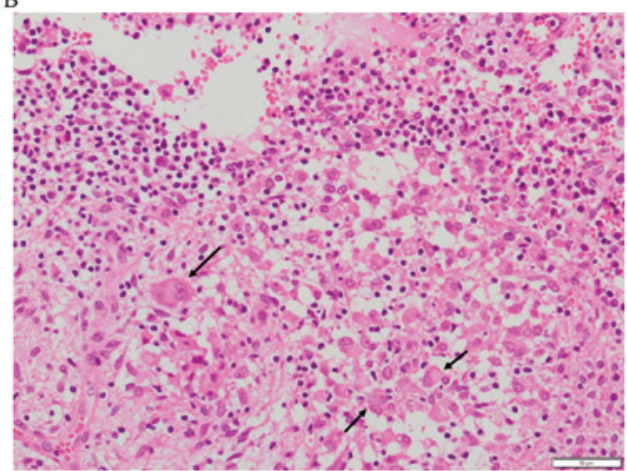

D

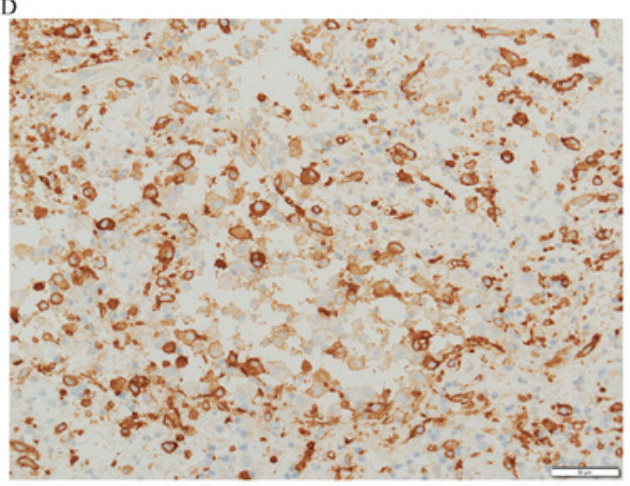

E

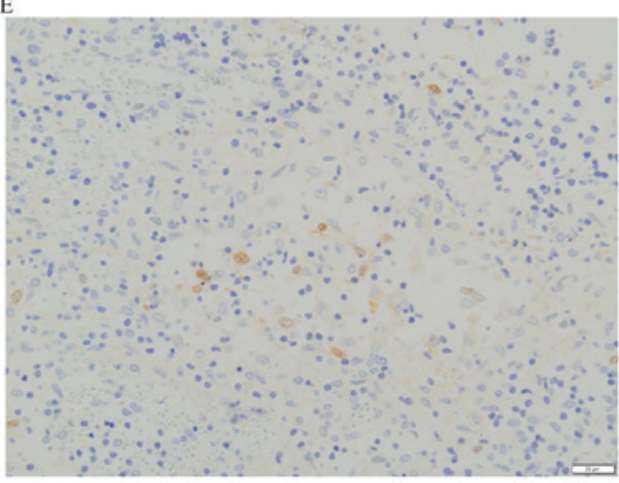

Figure 4. Microscopic findings. Large histiocytes demonstrated the emperipolesis of neutrophils, lymphocytes and plasma cells. Hematoxylin and eosin staining; (A) magnification, x100 and (B) magnification, x400. Emperipolesis was observed in the cytoplasm of the large histiocytes (arrow). Immunohistochemical findings revealed the positive staining for (C) CD68, (D) CD163 and (E) S-100. Magnification, $x 400$.

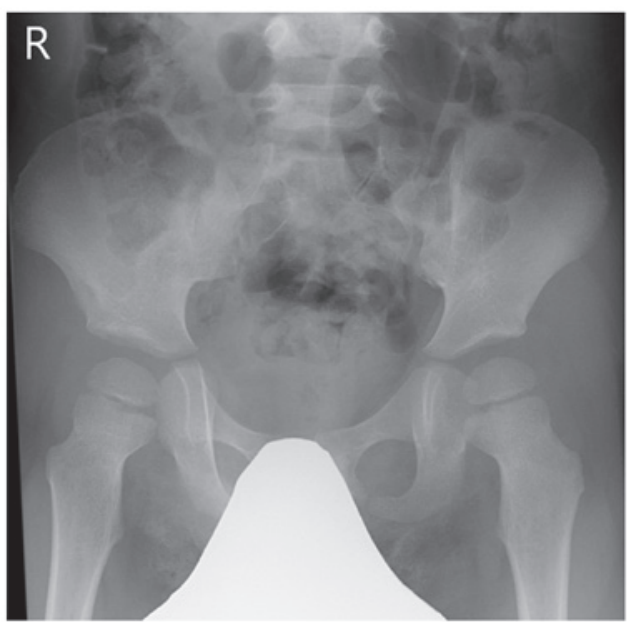

Figure 5. Plain radiograph after 18 months of follow-up. After 18 months of follow-up, plain radiography shows that the acetabular lytic lesion has disappeared. of RDD differentiates it from LCH, in which the histiocytes are CDla-positive and do not display emperipolesis. ECD can be differentially diagnosed from RDD based on the lack of emperipolesis, negative staining for S100 protein, and a characteristic imaging appearance that includes diaphyseal osteosclerosis of the long bone (4).

In the present case, microscopic findings showed emperipolesis within the histiocyte cytoplasm. Immunohistochemistry was positive for CD45, CD68, CD163, and lysozome and negative for CD1a. The patient had no clinical or laboratory evidence to support diagnoses of osteomyelitis, osteosarcoma, Ewing's sarcoma, or metastatic bone tumor, such as metastatic neuroblastoma or lymphoma. Thus, the diagnosis of primary bone RDD of the ilium was made.

Extranodal disease occurs in the upper respiratory tract, salivary glands, eyelids, and skin in approximately $28 \%$ of cases. Bone involvement in association with nodal disease is seen in $<10 \%$ of cases (2). Mosheimer et al (6) reviewed 


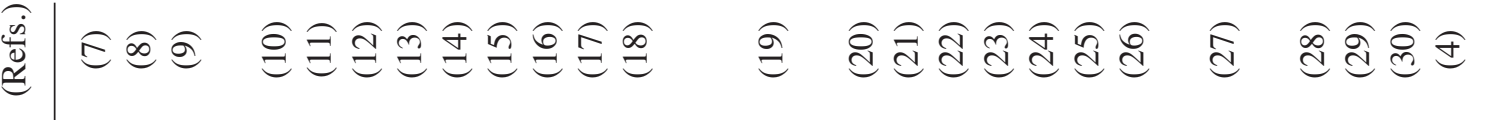

言

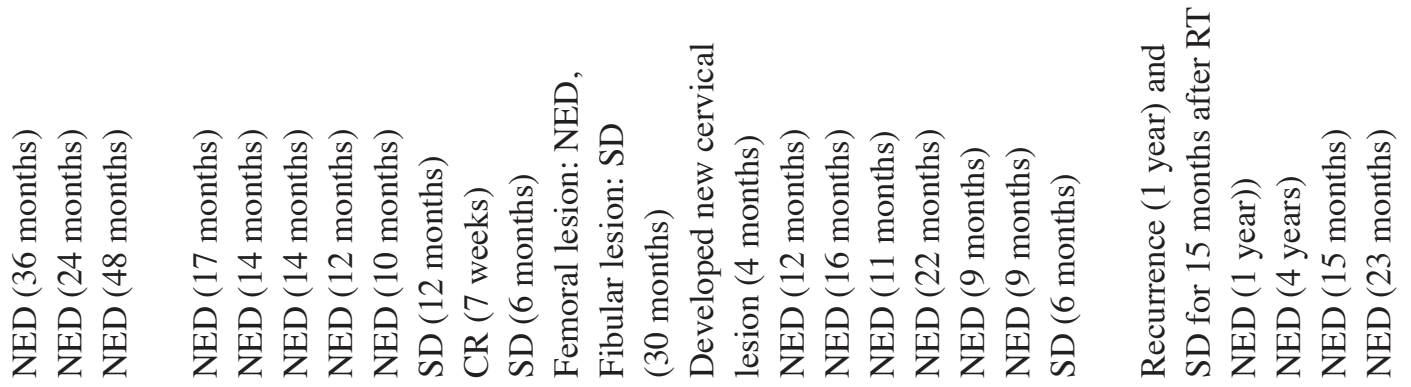

$\underline{\underline{t}}$

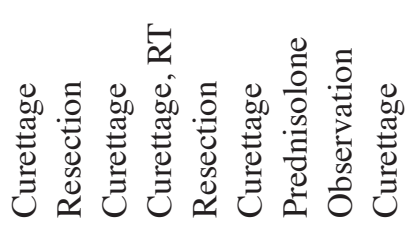

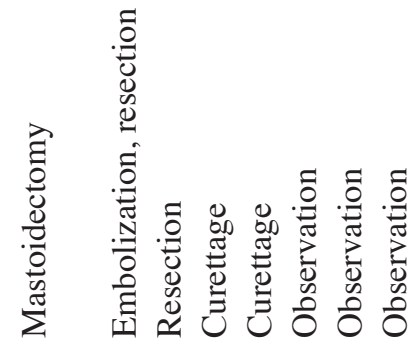

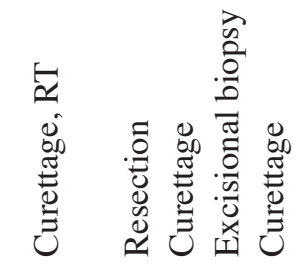

है

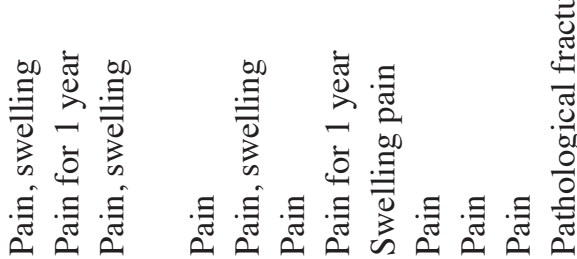

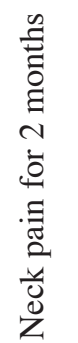

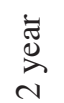

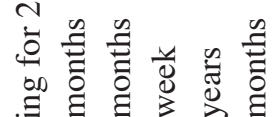

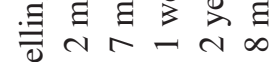

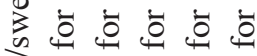

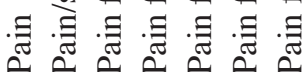

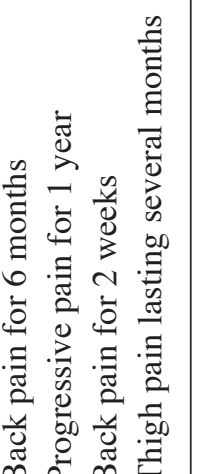

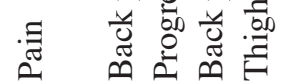

光

혼

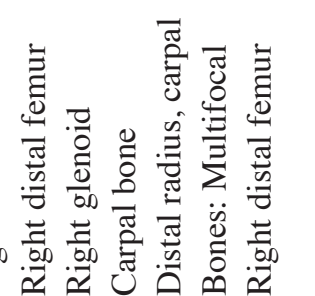

웅

苞

0
0
0
0
0
0
0
0

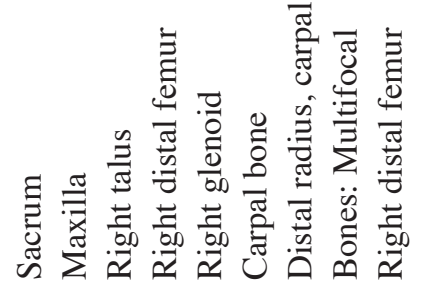

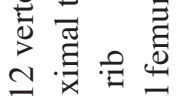

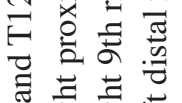

䒕

巧垊 $\Sigma$

$\Sigma$

巧巧巧 $\Sigma$ 巧

L

9

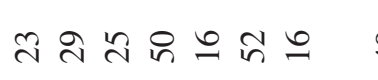

$\because \curvearrowleft \infty$

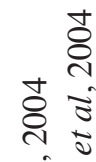

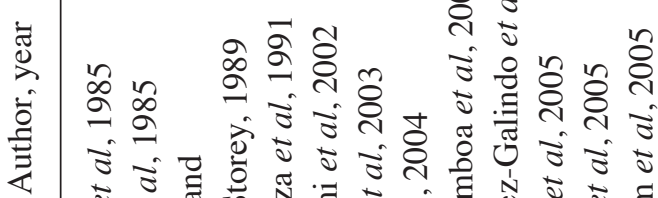

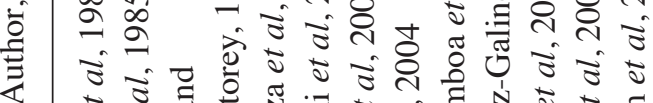

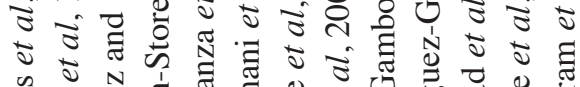

券

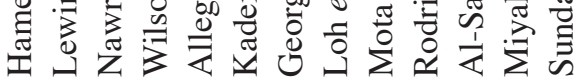

$\dot{\dot{z}} \mid-n m+n b-\infty a \varrho=\simeq$

9

ஓ्丶

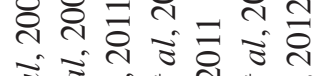

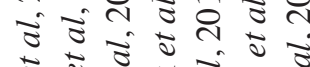

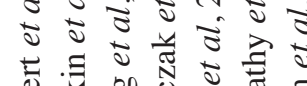

尝

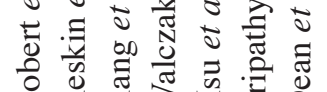

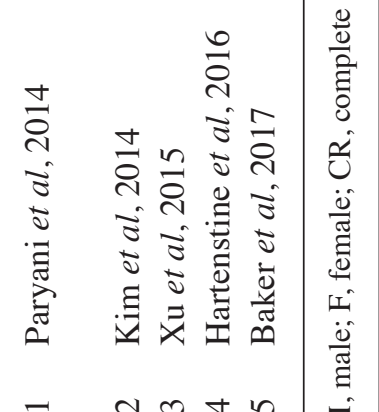


108 RDD patients with bone involvement and reported that primary RDD of the bone was observed in 67 (74.4\%) cases. Typical symptoms include pain and swelling, but bone lesions may be an incidental finding. Few reports have described the details of primary bone RDD (4,7-31). The largest series describes 15 cases of primary intraosseous RDD (31).

The clinical information of 25 previously reported cases of primary bone RDD are summarized in Table I. Of the 25 patients, 9 patients were male (36\%), and 16 were female (64\%). The mean age was 28.7 (range: 1.5-60) years old. The two-year-old boy in the present case is the second youngest patient to have a solitary bone lesion without lymphadenopathy. Treatment in most patients consisted of curettage $(n=12)$ or resection $(n=8)$. One patient underwent curettage followed by radiotherapy. Four patients were managed conservatively, and their condition was classified as stable disease. One patient $(n=9)$ received prednisone because his bone lesion was unresectable. Paryani reported a patient $(n=21)$ who underwent radiotherapy for recurrent disease after curettage for the primary lesion. Overall, the clinical outcome of primary intraosseous RDD is good. Curettage and resection are effective for achieving local control.

In the present case, the osteolytic lesion of the RDD showed spontaneous remission without residual bone deformity after curettage of the lesion at the incisional biopsy. However, previous reports indicated various treatment strategies, including corticosteroids, chemotherapy, radiotherapy, surgical curettage, and resection. Among all RDD patients, $20 \%$ show spontaneous remission without therapy (3). Mosheimer et al (6) reported that additional nodal manifestations of osseous RDD led to a more systemic treatment approach. Mostly intensive treatment was related to disease manifestations of problematic organs, including the central nervous system, vessels, orbit, and nasal cavity. Demicco et al (31) reported the clinical course of RDD of bone. Of 12 patients that were available for follow-up, 5 eventually developed additional extraosseous manifestations, including testicular, lymph node, and subcutaneous lesions. One patient developed additional multiple lesions of bone without extraosseous disease. These additional lesions developed from three months to three years after initial treatment. Thus, at least three years of follow-up may be necessary to detect the development of additional lesions.

RDD arising from bone has been reported in the literature. However, primary bone RDD without lymphadenopathy is extremely rare. Furthermore, the majority of patients are adolescents and young adults, and the mean age at the onset is 20 years old. There was only one report describe a patient under five years old. Before the biopsy, we suspected Langerhans cell histiocytosis as the differential diagnosis. It is important to consider primary RDD of bone as a differential diagnosis when osteolytic lesions are observed, even if the patient is under five years old.

\section{Acknowledgements}

The authors would like to thank Dr Oda Yoshinao, Professor of the Department of Anatomic Pathology, Pathological Sciences, Kyushu University (Fukuoka, Japan), for his advice regarding pathological diagnosis.

\section{Funding}

No funding was received.

\section{Availability of data and materials}

All data generated or analyzed during this study are included in this published article.

\section{Author's contributions}

YIz, KS, HK, YO and AM examined clinical findings, including laboratory data and radiographic images, and discussed the rsults. YIm pathologically diagnosed the patient. HK provided helpful advice due to their knowledge of bone diseases in childhood. YIz drafted the manuscript. AM takes full responsibility for the work as a whole, including the study design, access to data and the decision to submit and publish the manuscript. All authors read and approved the final manuscript.

\section{Ethics approval and consent to participate}

Clinical information was obtained by reviewing medical records. The study protocol was approved by the Ethics Committee of University of Fukui.

\section{Patient consent for publication}

Written informed consent for the publication of patient data/ images was obtained from the patient's parents.

\section{Competing interests}

The authors declare that they have no competing interests.

\section{References}

1. Rosai J and Dorfman RF: Sinus histiocytosis with massive lymphadenopathy: A newly recognized benign clinicopathological entity. Arch Pathol 87: 63-70, 1969.

2. Foucar E, Rosai J and Dorfman R: Sinus histiocytosis with massive lymphadenopathy (Rosai-Dorfman disease): Review of the entity. Semin Diagn Pathol 7: 19-73, 1990.

3. Dalia S, Sagatys E, Sokol L and Kubal T: Rosai-Dorfman disease: Tumor biology, clinical features, pathology, and treatment. Cancer Control 21: 322-327, 2014.

4. Baker JC, Kyriakos M, McDonald DJ and Rubin DA: Primary Rosai-Dorfman disease of the femur. Skeletal Radiol 46: 129-135, 2017.

5. Fletcher CD, Bridge JA, Hogendoorn PW and Mertens F (eds): WHO Classification of Tumours of the Soft Tissue and Bone. IARCPress, Lyon, p468, 2013.

6. Mosheimer BA, Oppl B, Zandieh S, Fillitz M, Keil F, Klaushofer K, Weiss $G$ and Zwerina J: Bone involvement in Rosai-Dorfman disease (RDD): A case report and systematic literature review. Curr Rheumatol Rep 19: 29, 2017.

7. Hamels J, Fiasse L and Thiery J: Atypical lymphohistiocytic bone tumor (osseous variant of Rosai-Dorfman disease?). Virchows Arch A Pathol Anat Histopatthol 408: 183-189, 1985.

8. Lewin JR, Das SK, Blumenthal BI, D'Cruz C, Patel RB and Howell GE: Osseous pseudotumor. Thesole manifestation of sinus histiocytosis with massive lymphadenopathy. Am J Clin Pathol 84: 547-550, 1985.

9. Nawoz IM and Wilson-Storey D: Sinus histiocytosis with massive lumphadenopathy (Rosai-Dorfman disease). Histopathology 14: 91-99, 1989. 
10. Allegranza A, Barbareschi M, Solero CL, Fornari M and Lasio G Primary lymphohistiocytic tumour of bone: A primary osseous localization of Rosai-Dorfman disease. Histpathology 18: 83-86, 1991.

11. Kademani D, Patel SG, Prasad ML, Huvos AG and Shah JP: Intraoral presentation of Rosai-Dorfman disease: A case report and review of the literature. Oral Surg Oral Med Oral Pathol Radiol Endod 93: 699-704, 2002.

12. George J, Stacy G, Peabody T and Montag A: Rosai-Dorfman disease manifesting as a solitary lesion of the radius in a 41-year-old woman. Skeletal Radiol 32: 236-239, 2003.

13. Loh SY, Tan KB, Wong YS and Lee YS: Rosai-Dorfman disease of the triquetrum without lymphadenopathy. A case report. J Bone Joint Surg Am 86: 595-598, 2004.

14. Mota Gamboa JD, Caleiras E and Rosas-Uribe A: Extranodal Rosai-Dorfman disease. Clinical and pathological characteristics in a patient with a pseudotumor of bone. Pathol Res Pract 200: 423-428, 2004

15. Rodriguez-Galindo C, Helton KJ, Sánchez ND, Rieman M, Jeng $M$ and Wang W: Extranodal Rosai-Dorfman disease in children. J Pediatr Hematol Oncol 26: 19-24, 2004.

16. Al-Saad K, Thorner P, Ngan BY, Gerstle JT, Kulkarni AV, Babyn P, Grant RM, Read S, Laxer RM and Chan HS: Extranodal Rosai-Dorfman disease with multifocal bone and epidural involvement causing recurrent spinal cord compression. Pediatr Dev Pathol 8: 593-598, 2005.

17. Miyake M, Tateisi U, Maeda T, Arai Y, Sugimura K and Hasegawa T: Extranodal Rosai-Dorfman disease: A solitary lesion with soft tissue reaction. Radiat Med 23: 439-442, 2005.

18. Sundaram C, Uppin Shantveer G, Chandrashekar P, Prasad VB and Umadevi M: Multifocal osseous involvement as the sole manifestation of Rosai-Dorfman disease. Skeletal Radiol 34: 658-664, 2005.

19. Tubbs RS, Kelly DR, Mroczek-Musulman EC, Hammers YA Berkow RL, Oakes WJ and Grabb PA: Spinal cord compression as a result of Rosai-Dorfman disease of the upper cervical spine in a child. Childs Nerv Syst 21: 951-954, 2005.

20. Robert EG, Fallon KB and Tender GC: Isolated Rosai-Dorfman disease of the sacrum. Case illustration. J Neurosurg Spine 4: $425,2006$.
21. Keskin A, Genc F and Günhan O: Rosai-Dorfman disease involving maxilla: A case report. J Oral Maxillofac Surg 65: 2563-2568, 2007.

22. Kang RW, McGill KC, Lin J and Gitelis S: Chronic Ankle pain and Swelling in a 25-year-old woman: An unusual case. Clin Orthop Relat Res 469: 1517-1521, 2011.

23. Walczak BE, Halperin DM, Bdeir RW and Irwin RB: Orthopaedic case of the month A 50-year-old woman with persistent knee pain. Clin Orthop Relat Res 469: 3527-3532, 2011.

24. Hsu AR, Bhatia S, Kang RW, Arvanitis L, Nicholson GP and Virkus WW: Extranodal Rosai-Dorfman disease presenting as an isolated glenoid lesion in a high school athlete. J Shoulder Elbow Surg 21: e6-e11, 2012.

25. Tripathy K, Misra A, Sahu AK and Patnaik K: Extranodal Rosai-Dorfamn disease in a carpal bone. Indian J Ortthop 46: 487-489, 2012

26. Dean EM, Wittig JC, Vilalobos C and Garcia RA: A 16-year-old boy with multifocal, painless osseous lesions. Clin Orthop Relat Res 470: 2640-2645, 2012.

27. Paryani NN, Daugherty LC, O'Connor MI and Jiang L: Extranodal Rosai-Dorfman disease of the bone treated with surgery and radiotherapy. Rare Tumors 6: 5531, 2014.

28. Kim DY, Park JH, Shin DA, Yi S, Ha Y, Yoon DH and Kim KN: Rosai-Dorfman disease in thoracic spine: A rare case of compression fracture. Korean J Spine 11: 198-201, 2014.

29. Xu J,Liu CH, Wang YS and Chen CX:Extranodal Rosai-Dorfaman disease as isolated lesion of the tibia diagnosed by fine-needle aspiration cytology. Medicine (Baltimore) 94: e2038, 2015.

30. Hartenstine J, Jackson H and Mortman K: A 38-year-old woman with an osteolytic rib lesion. Chest 149: e79-e85, 2016.

31. Demicco EG, Rosenberg AE, Bjornsson J, Rybak LD, Unni KK and Nielsen GP: Primary Rosai-Dorfman disease of bone: A clinicopathologic study of 15 cases. Am J Surg Pathol 34: 1324-1333, 2010

c) (i) $\odot$ This work is licensed under a Creative Commons C. ${ }_{\mathrm{EY}}$ No No Attribution-NonCommercial-NoDerivatives 4.0 International (CC BY-NC-ND 4.0) License. 